\title{
Relaxation of quantum states under energy perturbations
}

\author{
Dorje C. Brody*, Lane P. Hughston ${ }^{\dagger}$ and Joanna Syroka* \\ *Blackett Laboratory, Imperial College, London SW7 2BZ, UK \\ $\dagger$ Department of Mathematics, King's College London, Strand, London WC2R 2LS, UK
}

(October 23, 2018)

The energy-based stochastic extension of the Schrödinger equation is perhaps the simplest mathematically rigourous and physically plausible model for the reduction of the wave function. In this article we apply a new simulation methodology for the stochastic framework to analyse formulae for the dynamics of a particle confined to a square-well potential. We consider the situation when the width of the well is expanded instantaneously. Through this example we are able to illustrate in detail how a quantum system responds to an energy perturbation, and the mechanism, according to the stochastic evolutionary law, by which the system relaxes spontaneously into one of the stable eigenstates of the Hamiltonian. We examine in particular how the expectation value of the Hamiltonian and the probability distribution for the position of the particle change in time. An analytic expression for the typical timescale of relaxation is derived. We also consider the small perturbation limit, and discuss the relation between the stochastic framework and the quantum adiabatic theorem.

\section{INTRODUCTION}

Consider a quantum system for which the Hamiltonian undergoes a sudden change $\hat{h} \rightarrow$ $\hat{H}$ at time $t=0$ in such a manner that no mechanical 'work' is done on or by the system. Here $\hat{h}$ denotes the Hamiltonian before the change, and $\hat{H}$ denotes the Hamiltonian after the change.

If the initial wave function of the system is given by $\psi_{0}(x)$, the probability that the wave function $\psi_{t}(x)$ at a later time $t>0$ will be found in the $n$-th energy eigenstate $\chi_{n}(x)$ of the new Hamiltonian $\hat{H}$ is determined by the transition amplitude between $\psi_{0}(x)$ and $\chi_{n}(x)$. The assumption that no work is done on or by the system during the sudden change in the Hamiltonian implies that the expectation of the Hamiltonian is preserved under such a transformation. That is to say, the relation

$$
\int \psi_{0}^{*}(x) \hat{h} \psi_{0}(x) \mathrm{d} x=\int \psi_{t}^{*}(x) \hat{H} \psi_{t}(x) \mathrm{d} x,
$$

holds even though the Hamiltonian itself has changed.

Just after the application of the perturbation, the wave function can be represented by an expansion of $\psi_{0}(x)$ in terms of the eigenfunctions $\chi_{n}(x)$ of $\hat{H}$. The state then evolves according to the unitary law governed by $\hat{H}$ in such a way that the expectation value of $\hat{H}$ is preserved. According to the unitary law, the wave function $\psi_{t}(x)$ will remain in a state of superposition of the eigenmodes of $\hat{H}$, and this superposition will linger indefinitely in time. Indeed, it is generally necessary to append to quantum theory an additional postulate to the effect that only as a consequence of another kind of a sudden perturbation, namely, an 
operation of measurement, will the wave function of the system be able to 'jump' into one or another of the eigenstates of the new Hamiltonian.

In contrast, by use of the stochastic extension to the Schrödinger equation that we discuss in $\S 3$, it is possible to model the dynamics of the wave function in such a manner that, after the system is perturbed, the wave function spontaneously relaxes to one of the eigenstates of the new Hamiltonian. It is a remarkable fact that the probability laws thus arising from the stochastic dynamics give rise to statistical predictions that are essentially in agreement with the standard predictions of quantum theory.

The purpose of this paper is to present a detailed analysis of the phenomenon of stochastic relaxation in the simple case of a particle in a potential well when the width of the well is instantaneously enlarged. The structure of the paper is as follows. In $\S 2$ we review the basic setup for a free particle in a potential well when the well is subjected to a sudden expansion. We argue that standard quantum mechanics does not give a completely satisfactory account of the matter. In $\S 3$ we review the formalism of the standard energy-based stochastic extension of the Schrödinger equation, and propose the use of stochastic relaxation as a basis for the description of the dynamics of a quantum system following a perturbation. In $\S \S 4-5$ we construct the solution to the stochastic system and describe its properties, and in $\S \S 6-7$ we indicate how the solution can be used for the efficient construction of simulations. More precisely, we show that, according to the stochastic evolutionary law, the system energy fluctuates randomly and eventually relaxes to one of the eigenvalues of the new Hamiltonian. Graphic illustrations are provided for the behaviour of the energy, as well as the probability density of the location of the particle in the well. In $\S 8$ we present an analysis of the timescale associated with the eventuality of relaxation. This analysis then forms the basis of a discussion of a stochastic version of the quantum adiabatic theorem, presented in $\S \S 9-10$.

\section{FREE EXPANSION IN A POTENTIAL WELL}

We analyse here the sudden expansion of a one-dimensional potential well in which a particle of mass $\mu$ is trapped. Before the expansion, the width of the well is $L$. The energy spectrum of the particle is given by

$$
\epsilon_{n}=\frac{\pi^{2} \hbar^{2} n^{2}}{2 \mu L^{2}}
$$

where $n=1,2, \ldots, \infty$, for which the corresponding eigenfunctions are

$$
\phi_{n}(x)=\sqrt{\frac{2}{L}} \sin \left(\frac{n \pi}{L} x\right), \quad 0 \leq x \leq L .
$$

We assume that initially the particle is in one of the energy eigenstates associated with the potential.

Let us suppose that at $t=0$ the Hamiltonian $\hat{h}$ is changed in such a way that the width of the potential is increased from $L$ to $\alpha L$, where $\alpha \geq 1$. Then for any $t>0$ the wave function of the system can be expressed as a superposition of the normalised stationary states of the new Hamiltonian $\hat{H}$. These are given by 


$$
\chi_{n}(x)=\sqrt{\frac{2}{\alpha L}} \sin \left(\frac{n \pi}{\alpha L} x\right), \quad 0 \leq x \leq \alpha L,
$$

for which the associated eigenvalues are

$$
E_{n}=\frac{\pi^{2} \hbar^{2} n^{2}}{2 \mu \alpha^{2} L^{2}}
$$

where $n=1, \ldots, \infty$. It follows according to the unitary dynamics of the Schrödinger equation that after the expansion has taken place the system will be in an indefinite state of energy, and will remain so.

If the particle is initially in the $n$-th eigenstate $\phi_{n}(x)$ with energy $\epsilon_{n}$, then the probability $\pi_{n m}$ that, after the expansion has taken place, the particle will be found in the $m$-th eigenstate $\chi_{m}(x)$ of the new Hamiltonian, with energy $E_{m}$, is

$$
\pi_{n m}=\left(\int_{0}^{L} \phi_{n}(x) \chi_{m}(x) \mathrm{d} x\right)^{2}
$$

A short calculation shows that

$$
\pi_{n m}=\frac{4 \alpha^{3} n^{2}}{\pi^{2}\left(m^{2}-\alpha^{2} n^{2}\right)^{2}} \sin ^{2}\left(\frac{\pi m}{\alpha}\right) .
$$

Clearly, if such a change of state occurs, then energy is not strictly conserved. The conservation of energy is maintained in expectation, however. Indeed, we have the identity

$$
\sum_{m=1}^{\infty} \pi_{n m} E_{m}=\epsilon_{n}
$$

which is valid for all $n$ and for all $\alpha$, by virtue of which we can confirm that the expectation value of the Hamiltonian is a constant of the motion (Bender, Brody \& Meister 1999).

To understand the energy conservation law intuitively in this example, we can regard the potential walls as forming the 'piston' of a one-dimensional cylinder. Suppose we consider an ensemble consisting of a large number of independent identical particles in the cylinder. Then when the piston is instantaneously moved outwards, the system undergoes a free expansion. During such a process, the particles do no mechanical work on the piston. As a result, there is no net flow of energy going out of the cylinder, and thus the energy is conserved. The 'energy' that is conserved in this case is the ensemble energy, i.e. the product of total number of particles and the expectation value of the energy of an individual particle.

The foregoing formulae follow directly from the basic principles of quantum theory. In particular, the standard interpretation of quantum theory maintains that after the expansion the system will remain in an indefinite state of energy, until the energy is measured. However, there are many situations in which it is natural to presume that after the passage of some time the system spontaneously relaxes into one or another of the eigenstates of the new Hamiltonian, irrespective of whether a measurement is made. Many natural phenomena are of this character: after perturbation, there follows relaxation. Quantum theory, as such, does not account for this satisfactorily. There is arguably an implicit assumption that, in 
the absence of specific acts of measurement, quantum systems of any significant size or complexity will settle into a stable eigenstate, typically an eigenstate of energy.

In what follows we take the point of view that, after perturbation, the system does eventually evolve spontaneously into one of the stable eigenstates. Quantum theory in itself offers no clue as regards the precise mechanism according to which the system relaxes to the new stationary state. These questions can, nevertheless, be addressed in a very satisfactory way by use of the dynamics of the stochastic extension of the Schrödinger equation, as we shall demonstrate.

\section{ENERGY-BASED STOCHASTIC DYNAMICS}

In what follows we shall suppose that the dynamical mechanism governing the relaxation of the quantum system is determined by the standard energy-based stochastic extension of the Schrödinger equation. This is given by the following stochastic differential equation of the Ito type:

$$
\mathrm{d} \psi_{t}(x)=-\mathrm{i} \hat{H} \psi_{t}(x) \mathrm{d} t-\frac{1}{8} \sigma^{2}\left(\hat{H}-H_{t}\right)^{2} \psi_{t}(x) \mathrm{d} t+\frac{1}{2} \sigma\left(\hat{H}-H_{t}\right) \psi_{t}(x) \mathrm{d} W_{t}
$$

for which it is assumed that there is a prescribed initial wave function $\psi_{0}(x)$. Here $W_{t}$ denotes a standard Wiener process, and

$$
H_{t}=\frac{\int \psi_{t}^{*}(x) \hat{H} \psi_{t}(x) \mathrm{d} x}{\int \psi_{t}^{*}(x) \psi_{t}(x) \mathrm{d} x}
$$

is the random process corresponding to the expectation value of the Hamiltonian operator $\hat{H}$ in the random state $\psi_{t}(x)$. The volatility parameter $\sigma$ appearing in (9) has the units

$$
[\sigma]=[\text { Energy }]^{-1}[\text { Time }]^{-1 / 2} .
$$

Dynamical equations of the type (9) for the evolution of the wave function and various generalisations thereof, were introduced originally as simple models to characterise the collapse of the wave function when a measurement is carried out on a system (Gisin 1984, 1989, Ghirardi, et al 1986, 1990, Diosi 1988; see, e.g., Percival 1998, Pearle 2000 and Bassi \& Ghirardi 2002 and references cited therein for a more comprehensive account of the relevant literature). The idea that wave function should proceed to energy eigenstates was proposed by Bedford and Wang $(1975,1977)$. The specific energy-based form of the dynamics (9), which has been studied by Gisin 1989, Percival 1994, 1995, Hughston 1996, Adler \& Horowitz 2000, Adler 2002, and Brody \& Hughston 2002, amongst others, is the most parsimonious of these state reduction models and in many respects perhaps the most attractive as the basis for a fundamental model. In this paper we carry the physical application of the stochastic theory a step further and propose the use of (9) as an elementary model for characterising the relaxation of the state of a quantum system when its Hamiltonian has been perturbed. We shall leave open here the question of whether the volatility parameter $\sigma$ governing the timescale of relaxation is phenomenological in character, i.e. varying according to the structure of the system, or universal, e.g., Planckian. Before deriving the solution to (9) and applying the results to perturbation theory, we shall briefly sketch some of the basic mathematical and 
physical properties associated with the dynamics (9). For further details, see Adler et al. (2001) and references cited therein.

Let us first note that the coefficient of the Brownian motion in the third term of the righthand side of (9) is given by the difference of the Hamiltonian operator and its expectation, acting on the wave function. Thus if the system enters into an eigenstate of the Hamiltonian, this coefficient becomes zero, and the random fluctuations generated by the process $W_{t}$ make no further contribution to the dynamics of $\psi_{t}(x)$. This property likewise applies to the second term, which together with the third term 'drives' the system into a state of lower energy uncertainty. Starting from an arbitrary initial state, the system is randomly driven into states with lower energy variance, until it finally reaches an eigenstate of the Hamiltonian, in which the variance vanishes.

A process for which both of the coefficients of the $\mathrm{d} t$ term and the $\mathrm{d} W_{t}$ term in the associated stochastic differential equation are smooth functions of the process $\psi_{t}(x)$ itself is called a diffusion. If the number of Brownian motions is smaller than the dimensionality of the process $\psi_{t}(x)$, then the process is said to be a degenerate diffusion. In the present consideration, while the wave function $\psi_{t}(x)$ is an element of an infinite dimensional Hilbert space, there is only a single Brownian motion driving the system, and thus the dynamical equation (9) represents a highly degenerate diffusion. A typical feature of a degenerate diffusion, as opposed to a generic diffusion, is that it may have a 'focusing' effect. Indeed, in the case of (9) the wave function is focussed to one of the energy eigenstates, as a consequence of the dynamics.

Given the dynamical equation (9) for the wave function and the corresponding process (10) for the expected energy, we can determine the stochastic equation satisfied by $H_{t}$. Specifically, this is given by

$$
\mathrm{d} H_{t}=\sigma V_{t} \mathrm{~d} W_{t},
$$

where

$$
V_{t}=\frac{\left\langle\psi_{t}\left|\left(\hat{H}-H_{t}\right)^{2}\right| \psi_{t}\right\rangle}{\left\langle\psi_{t} \mid \psi_{t}\right\rangle}
$$

is the process associated with the variance of the energy. The variance process satisfies

$$
\mathrm{d} V_{t}=-\sigma^{2} V_{t}^{2} \mathrm{~d} t+\sigma \beta_{t} \mathrm{~d} W_{t},
$$

where

$$
\beta_{t}=\frac{\left\langle\psi_{t}\left|\left(\hat{H}-H_{t}\right)^{3}\right| \psi_{t}\right\rangle}{\left\langle\psi_{t} \mid \psi_{t}\right\rangle}
$$

is the third central moment of the energy. As a consequence, integrating (12) and (14) we find that the energy process can be expressed in the form

$$
H_{t}=H_{0}+\sigma \int_{0}^{t} V_{s} \mathrm{~d} W_{s}
$$

and the variance process can be written as 


$$
V_{t}=V_{0}-\sigma^{2} \int_{0}^{t} V_{s}^{2} \mathrm{~d} s+\sigma \int_{0}^{t} \beta_{s} \mathrm{~d} W_{s}
$$

Owing to elementary properties of the stochastic integrals (16) and (17) one can determine the relations

$$
\mathbb{E}\left[H_{u} \mid\left\{H_{s}\right\}_{0 \leq s \leq t}\right]=H_{t}
$$

and

$$
\mathbb{E}\left[V_{u} \mid\left\{V_{s}\right\}_{0 \leq s \leq t}\right] \leq V_{t}
$$

for $t \leq u$. Here $\mathbb{E}\left[\cdots \mid\left\{X_{s}\right\}_{0 \leq s \leq t}\right]$ denotes the conditional expectation given the history of the process $X_{s}$ from time 0 up to time $t$. Therefore, the conditional expectation of the energy process at any time $u \geq t$, given its history up to time $t$, is given by its value at time $t$. We thus say that $H_{t}$ satisfies the martingale condition. It follows that $H_{t}$ is on average conserved, whereas the variance $V_{t}$ tends, on average, to decrease, corresponding to the spontaneous reduction of the system to an eigenstate. The reductive character of the dynamics (9) is indicated by the fact that $V_{t}$ satisfies the supermartingale condition.

\section{ANALYTIC SOLUTION TO THE STOCHASTIC EQUATION}

Despite its nonlinearity, the dynamical equation (9) can be solved exactly to yield an analytic solution that fully characterises the dynamical state of the system at any time in terms of a pair of state variables (Brody \& Hughston 2002). The method of obtaining the solution, which is of some interest in its own right, makes use of the classical techniques of nonlinear filtering theory. More precisely, we consider a process of the form

$$
\xi_{t}=\sigma t H+B_{t}
$$

where $B_{t}$ is a Brownian motion, and $\sigma$ is the parameter that ultimately determines the characteristic relaxation timescale. The random variable $H$ takes on the value $E_{m}$ with probability $\pi_{m}$, where $\pi_{m}$ is taken to be the transition probability from the initial quantum state to the energy eigenstate with energy $E_{m}$. Intuitively, one can think of the process $\xi_{t}$ as representing the value of a phase, scaled by the constant $\sigma$, together with a random noise term, whose strength, relative to the variable $H$, decreases inverse-proportionally in time.

The random variable $H$ is to be thought of as representing the value of the energy to which the system ultimately relaxes after the passage of sufficient time. Given the trajectory of the process $\xi_{t}$ up to time $t$, we would like to determine the best estimate for the value of $H$. Because the standard error in the value of $B_{t}$ grows only like the square-root of time, it follows that as time passes the true value of $H$ is gradually revealed. The estimate will be denoted $H_{t}$, which is obtained by taking the conditional expectation of the random variable $H$, given the history of $\xi_{t}$ up to that time. Because $\xi_{t}$ is a Markov process, this implies that $H_{t}$ is the expectation of $H$ conditional on the value $\xi_{t}$, that is,

$$
H_{t}=\mathbb{E}\left[H \mid \xi_{t}\right] .
$$


The argument that $H_{t}$ is the best estimate for $H$ given the history of $\xi_{t}$ is as follows. Suppose $Y_{t}$ is any process that at time $t$ can be expressed as a functional of the history of $\xi_{s}$ for $0 \leq s \leq t$. Then the choice of $Y_{t}$ that minimises the expected mean square error $\mathbb{E}\left[\left(H-Y_{t}\right)^{2} \mid\left\{\xi_{s}\right\}_{0 \leq s \leq t}\right]$ given the history of $\xi_{t}$ is the process $H_{t}$ defined by (21). This can be deduced by a straightforward variational argument.

Because $H_{t}$ is given, for each value of $t$, by a conditional expectation with respect to the random variable $\xi_{t}$, it follows that $H_{t}$ can be expressed as a function of $\xi_{t}$. In order to determine the conditional expectation (21) more explicitly, we require the conditional probability $\mathbb{P}\left(H=E_{m} \mid \xi_{t}\right)$ for the random variable $H$. By use of the Bayes law for conditional probability, we find that this is given by

$$
\mathbb{P}\left(H=E_{m} \mid \xi_{t}\right)=\frac{\pi_{m} \rho\left(\xi_{t} \mid H=E_{m}\right)}{\sum_{n=1}^{\infty} \pi_{n} \rho\left(\xi_{t} \mid H=E_{n}\right)} .
$$

Here $\rho\left(\xi_{t} \mid H=E_{m}\right)$ denotes the conditional density function for the continuous random variable $\xi_{t}$ given that $H=E_{m}$. In deriving (22) we make use of the fact that the unconditional probability $\mathbb{P}\left(H=E_{m}\right)$ is just $\pi_{m}$ for the given initial state. We have also used the relation

$$
\rho\left(\xi_{t}\right)=\sum_{n=1}^{\infty} \pi_{n} \rho\left(\xi_{t} \mid H=E_{n}\right) .
$$

Since $B_{t}$ is a Brownian motion, it is by definition normally distributed with mean zero and variance $t$. Thus the conditional probability density for $\xi_{t}$ is

$$
\rho\left(\xi_{t} \mid H=E_{m}\right)=\frac{1}{\sqrt{2 \pi t}} \exp \left(-\frac{1}{2 t}\left(\xi_{t}-\sigma E_{m} t\right)^{2}\right) .
$$

Inserting this expression into (22), we deduce that

$$
\mathbb{P}\left(H=E_{m} \mid \xi_{t}\right)=\frac{\pi_{m} \exp \left(\sigma E_{m} \xi_{t}-\frac{1}{2} \sigma^{2} E_{m}^{2} t\right)}{\sum_{n=1}^{\infty} \pi_{n} \exp \left(\sigma E_{n} \xi_{t}-\frac{1}{2} \sigma^{2} E_{n}^{2} t\right)} .
$$

Since the energy process is given by the expectation

$$
H_{t}=\sum_{m=1}^{\infty} E_{m} \mathbb{P}\left(H=E_{m} \mid \xi_{t}\right)
$$

it follows that

$$
H_{t}=\frac{\sum_{m=1}^{\infty} \pi_{m} E_{m} \exp \left(\sigma E_{m} \xi_{t}-\frac{1}{2} \sigma^{2} E_{m}^{2} t\right)}{\sum_{m=1}^{\infty} \pi_{m} \exp \left(\sigma E_{m} \xi_{t}-\frac{1}{2} \sigma^{2} E_{m}^{2} t\right)} .
$$

We note that in (27) the process $H_{t}$ for the conditional expectation of the random variable $H$ is expressed in terms of a function of $t$ and $\xi_{t}$. As a consequence, the dynamics of the process $\xi_{t}$ can be expressed as a diffusion equation of the form

$$
\mathrm{d} \xi_{t}=\sigma H_{t} \mathrm{~d} t+\mathrm{d} W_{t}
$$

where $W_{t}$ is a standard Brownian motion with respect to the filtration generated by the history of $H_{t}$. The existence of a Brownian motion $W_{t}$ satisfying (28) follows as a consequence of well-established line of argument in nonlinear filtering theory (see Liptser \& Shiryaev 1974), the details of which in the present context are set out in Brody \& Hughston (2002). 


\section{STOCHASTIC RELAXATION}

In order to see directly the relaxation of the Hamiltonian process (27) to one of the energy eigenvalues, we can argue as follows.

Let us suppose that the random variable $H$ happens to take the value $E_{j}$. By definition such an event occurs with probability $\pi_{j}$. More precisely, we condition on the outcome of the event $H=E_{j}$ and analyse the evolution of process (27). Then, writing $\xi_{t}=\sigma E_{j} t+B_{t}$, we obtain, for the corresponding realisation of $H_{t}$, the expression

$$
H_{t}^{j}=\frac{\pi_{j} E_{j}+\sum_{m \neq j}^{\infty} \pi_{m} E_{m} \exp \left(\sigma\left(E_{m}-E_{j}\right) B_{t}-\frac{1}{2} \sigma^{2}\left(E_{m}-E_{j}\right)^{2} t\right)}{\pi_{j}+\sum_{m \neq j}^{\infty} \pi_{m} \exp \left(\sigma\left(E_{m}-E_{j}\right) B_{t}-\frac{1}{2} \sigma^{2}\left(E_{m}-E_{j}\right)^{2} t\right)}
$$

where the superscript $j$ in $H_{t}^{j}$ indicates the conditioning on the event $\left\{H=E_{j}\right\}$.

Because the exponential terms appearing in both denominator and numerator have the property that, as $t \rightarrow \infty$, the probability that these terms remain positive approaches zero, it follows that the energy process $H_{t}^{j}$ converges asymptotically to the designated eigenvalue $E_{j}$.

Indeed for any normally distributed random variable $B_{t}$ with mean zero and variance $t$ it is an elementary fact that if $\nu \neq 0$ then

$$
\lim _{t \rightarrow \infty} \mathbb{P}\left(\exp \left(\nu B_{t}-\frac{1}{2} \nu^{2} t\right)>x\right)=0
$$

for any given $x>0$.

The exact, closed-form solution (29) shows how the expectation value of the system Hamiltonian evolves in time. In particular, it shows how the system organises itself spontaneously to move into a stable, stationary state of the new Hamiltonian. Since formula (29) is expressed in terms of a simple analytic function of the Brownian motion $B_{t}$ and time $t$, there arises the possibility of efficiently simulating the evolution of the process $H_{t}$.

\section{WAVE FUNCTION DYNAMICS}

We return now to the analysis of the dynamics of the state $\psi_{t}(x)$ of a particle trapped in a potential well following a sudden expansion of the width of the well. In this case the solution to the stochastic differential equation (9) can be expressed in the form

$$
\psi_{t}(x)=\frac{\sum_{m=1}^{\infty} \pi_{m}^{1 / 2} \exp \left(-\mathrm{i} E_{m} t+\frac{1}{2} \sigma E_{m} \xi_{t}-\frac{1}{4} \sigma^{2} E_{m}^{2} t\right) \chi_{m}(x)}{\left(\sum_{m=1}^{\infty} \pi_{m} \exp \left(\sigma E_{m} \xi_{t}-\frac{1}{2} \sigma^{2} E_{m}^{2} t\right)\right)^{1 / 2}}
$$

Here $\chi_{m}(x)$ denotes the normalised eigenfunction of the Hamiltonian $\hat{H}$ with energy $E_{m}$, and the choice of the initial wave function $\psi_{0}(x)$ is implicit in the probability $\pi_{m}$ :

$$
\pi_{n m}=\left(\int_{0}^{L} \psi_{0}(x) \chi_{m}(x) \mathrm{d} x\right)^{2}
$$

The solution (31) can be verified by taking the stochastic differential of the right hand side of (31) and using the Ito rules, and then making the substitution (28). 
The convergence of the infinite sum in the numerator and the denominator of (31) for finite $t$ may not be immediately evident. In order to check that these summations converge, we substitute a particular realisation of the path for $\xi_{t}$, say, $\xi_{t}=\sigma E_{j} t+B_{t}$, when the random variable $H$ happens to take the value $E_{j}$. Then on account of the fact that $E_{m} \propto m^{2}$, we find that the summands decay, for each fixed $j$, like $\sim \exp \left(-m^{4}\right)$, and convergence is ensured.

Let us now consider the random dynamics of the probability density function

$$
\rho_{t}(x)=\psi_{t}^{*}(x) \psi_{t}(x)
$$

for finding the particle at the location $x$ in the interval $[0, \alpha L]$. We would like to obtain the probability distribution for the particle in the case where the final state of the system is given by the eigenstate $\chi_{j}(x)$ for some given value of $j$. The probability for this particular realisation to occur is given by $\pi_{j}$. By rearrangement of terms, and writing

$$
\omega_{m j}=E_{m}-E_{j}
$$

for the energy-level difference, we obtain

$$
\rho_{t}^{j}(x)=\frac{\left|\sum_{m} \pi_{m}^{1 / 2} \exp \left(-\mathrm{i} \omega_{m j} t+\frac{1}{2} \sigma \omega_{m j} B_{t}-\frac{1}{4} \sigma^{2} \omega_{m j}^{2} t\right) \chi_{m}(x)\right|^{2}}{\sum_{m} \pi_{m} \exp \left(\sigma \omega_{m j} B_{t}-\frac{1}{2} \sigma^{2} \omega_{m j}^{2} t\right)}
$$

for the probability density. Thus $\rho_{t}(x)$ is a measure-valued process, i.e. at each given time $t$ it is a smooth density function over $[0, \alpha L]$, but the form of the function evolves randomly in time until it relaxes to the final distribution $\chi_{j}^{2}(x)$.

\section{SIMULATION OF THE ENERGY AND THE PROBABILITY DENSITY}

\section{A version of the paper with figures can be downloaded from}

$$
\text { http://theory.ic.ac.uk/ brody/DCB/sa8.pdf }
$$

FIG. 1. Several realisations of the process $H_{t}$ for the expectation value of the Hamiltonian are illustrated. The initial condition is chosen to be $\psi_{0}(x)=\phi_{1}(x)$ and the final conditions are given by $\psi_{\infty}(x)=\chi_{1}(x), \chi_{2}(x), \chi_{3}(x), \chi_{4}(x), \chi_{5}(x), \chi_{6}(x)$, respectively. The horizontal lines indicate the energy levels $E_{j}$, where we have set $\alpha=2.5$. For convenience the vertical axis is expressed in units of the characteristic energy $\varepsilon=\hbar^{2} / 2 \mu L^{2}$, and the horizontal axis is expressed in units of the corresponding characteristic time interval $1 / \sigma^{2} \varepsilon^{2}$. When $j=5$, we have $\pi_{5}=0$. As a result, if $E_{j}=E_{5}$ is chosen for the value of the random variable, the energy does not reduce to that eigenvalue. 
With these formulae at hand, we now consider the simulation of the random trajectory for the expectation value of the Hamiltonian governed by the dynamics (27). We also consider the simulation of the corresponding probability density function (35) for the position of the particle in the potential well. The quantum system is defined by specifying the value of the mass $\mu$ of the particle, the width $L$ of the well, the volatility parameter $\sigma$ governing the stochastic dynamics, and the well expansion factor $\alpha$. For simplicity, we shall examine the case where the system is initially in its ground state $\phi_{1}(x)$, although any other initial condition, including mixed initial states, can be treated analogously. Then the energy process (29), corresponding to the particular realisation $\psi_{\infty}(x)=\chi_{j}(x)$ of the terminal state, can be written in the form

$$
H_{t}^{j}=\frac{\sum_{m} \pi_{m} E_{m} \exp \left(\sigma \omega_{m j} B_{t}-\frac{1}{2} \sigma^{2} \omega_{m j}^{2} t\right)}{\sum_{m} \pi_{m} \exp \left(\sigma \omega_{m j} B_{t}-\frac{1}{2} \sigma^{2} \omega_{m j}^{2} t\right)} .
$$

The associated probability density for the position of the particle in the interval $[0, \alpha L]$ is given by

$$
\begin{aligned}
\rho_{t}^{j}(x)= & \frac{\left(\sum_{m} \pi_{m}^{1 / 2} \exp \left(\frac{1}{2} \sigma \omega_{m j} B_{t}-\frac{1}{4} \sigma^{2} \omega_{m j}^{2} t\right) \chi_{m}(x) \cos \left(\omega_{m j} t\right)\right)^{2}}{\sum_{m} \pi_{m} \exp \left(\sigma \omega_{m j} B_{t}-\frac{1}{2} \sigma^{2} \omega_{m j}^{2} t\right)} \\
& +\frac{\left(\sum_{m} \pi_{m}^{1 / 2} \exp \left(\frac{1}{2} \sigma \omega_{m j} B_{t}-\frac{1}{4} \sigma^{2} \omega_{m j}^{2} t\right) \chi_{m}(x) \sin \left(\omega_{m j} t\right)\right)^{2}}{\sum_{m} \pi_{m} \exp \left(\sigma \omega_{m j} B_{t}-\frac{1}{2} \sigma^{2} \omega_{m j}^{2} t\right)} .
\end{aligned}
$$

\title{
A version of the paper with figures can be downloaded from
}

\author{
http://theory.ic.ac.uk/ brody/DCB/sa8.pdf
}

FIG. 2. Several realisations of the probability distributions $\rho_{t}(x)$ for the particle in the well are illustrated. The initial and final conditions are $\psi_{0}(x)=\phi_{1}(x)$ and $\psi_{\infty}(x)=\chi_{2}(x)$, respectively, and we set $\alpha=2.5$. The $x$-axis is measured in units of $L$, and the time axis is measured in units of $4 \mu^{2} L^{4} / \hbar^{4} \sigma^{2}$. Most realisations have the property that the initial state swiftly relaxes into the terminal state.

The process $H_{t}^{j}$ defined by (36) can be thought of as a kind of 'Brownian bridge' that interpolates between the two energy levels $\epsilon_{1}$ and $E_{j}$. That is to say, initially the system has energy $\epsilon_{1}$, and then it progresses along a random trajectory to reach the designated terminal level $E_{j}$. A similar remark applies to the density process $\rho_{t}^{j}(x)$ of (37), which interpolates 
between the two functions $\phi_{1}^{2}(x)$ and $\chi_{j}^{2}(x)$. It should be evident that, unlike $H_{t}$, the process $H_{t}^{j}$ does not conserve energy.

To obtain a better feeling for the dynamics of $H_{t}^{j}$ it will be useful to examine the associated stochastic differential equation. In particular, if we take the stochastic differential of (36), then after a rearrangement of terms we obtain

$$
\mathrm{d} H_{t}^{j}=\sigma^{2} V_{t}^{j}\left(E_{j}-H_{t}^{j}\right) \mathrm{d} t+\sigma V_{t}^{j} \mathrm{~d} B_{t}
$$

where the nonnegative process $V_{t}^{j}$ is given by

$$
V_{t}^{j}=\frac{\sum_{m} \pi_{m}\left(E_{m}-H_{t}^{j}\right)^{2} \exp \left(\sigma \omega_{m j} B_{t}-\frac{1}{2} \sigma^{2} \omega_{m j}^{2} t\right)}{\sum_{k} \pi_{m} \exp \left(\sigma \omega_{m j} B_{t}-\frac{1}{2} \sigma^{2} \omega_{m j}^{2} t\right)} .
$$

We learn from (38) that $H_{t}^{j}$ is a mean-reverting process with mean level $E_{j}$ and reversion rate $\sigma^{2} V_{t}^{j}$. In fact, one can integrate (38) by means of the standard technique used in the case of the classical Ornstein-Uhlenbeck process (see, e.g., Doob 1942) to obtain

$$
H_{t}^{j}=E_{j}+\left(H_{0}^{j}-E_{j}\right) \mathrm{e}^{-\sigma^{2} \int_{0}^{t} V_{s}^{j} \mathrm{~d} s}+\sigma \int_{0}^{t} \mathrm{e}^{-\sigma^{2} \int_{u}^{t} V_{s}^{j} \mathrm{~d} s} V_{u}^{j} \mathrm{~d} B_{u}
$$

which shows clearly how the information of the initial condition is damped away at the rate $\sigma^{2} \bar{V}_{t}^{j}$, where

$$
\bar{V}_{t}^{j}=\frac{1}{t} \int_{0}^{t} V_{s}^{j} \mathrm{~d} s
$$

Alternatively, we can write (40) in the form

$$
H_{t}^{j}-E_{j}=\left(H_{0}^{j}-E_{j}+\sigma \int_{0}^{t} \mathrm{e}^{\sigma^{2} \int_{0}^{u} V_{s}^{j} \mathrm{~d} s} V_{u}^{j} \mathrm{~d} B_{u}\right) \mathrm{e}^{-\sigma^{2} \int_{0}^{t} V_{s}^{j} \mathrm{~d} s},
$$

which expresses the difference $H_{t}^{j}-E_{j}$ between $H_{t}^{j}$ and its terminal value as the product of a martingale and a positive decreasing process.

With expressions (36) and (37) at hand we proceed to simulate some realisations of these processes. For convenience we take advantage of the fact that there is a natural energy unit $\varepsilon$ determined by the problem, which is given by

$$
\varepsilon=\frac{\hbar^{2}}{2 \mu L^{2}}
$$

Thus, when we plot figures we can express energies in units of $\varepsilon$, and we can express times in units of

$$
\frac{1}{\sigma^{2} \varepsilon^{2}}=\frac{4 \mu^{2} L^{4}}{\hbar^{4} \sigma^{2}}
$$

In the analysis that follows, energy and time will be expressed in these units.

In Figure 1 the energy process $H_{t}^{j}$ is shown for several values of $j$ ranging from 1 to 6 , where initially the system is in the ground state of the old potential, with energy $\epsilon_{1}=\pi^{2}$. In 
these examples the width of the potential is expanded at $t=0$ by a factor of $\alpha=2.5$. The most likely transition to occur, given the initial state $\phi_{1}(x)$, when the width is expanded by a factor of 2.5, is the first excited state $\chi_{2}(x)$. In this example it follows as a consequence of (匹) that $\pi_{15}=0$, and hence the transition into the fifth energy level does not occur. Instead, there is a transition to the fourth level.

A version of the paper with figures can be downloaded from

http://theory.ic.ac.uk/ brody/DCB/sa8.pdf

FIG. 3. The expectation of $H_{t}^{j}$ is shown for a variety of realisations $j=1,2,3,4,5,6$, when the initial state is $\phi_{1}(x)$. The curious behaviour for $j=5$ arises in this example because $\pi_{5}=0$, and the state never collapses to $\chi_{5}(x)$. Instead, the state reduced to the eigenstate having the energy closest to $E_{5}$, which in the present case is the fourth eigenstate.

Several realisations of the corresponding probability distribution for the particle are shown in Figure 2, where the terminal wave function is the first excited state $\chi_{2}(x)$. The probability of this event to be realised is as large as $\sim 0.43$. In most examples the initial state swiftly changes into the terminal state, although some interesting behaviour can occasionally be observed when the value of the Brownian motion $B_{t}$ grows large.

In Figure 3 the numerical average of the energy process, corresponding to the expectation of $H_{t}^{j}$, is shown for a range of terminal states in the case where the expansion factor is $\alpha=2.5$. Each graph represents the average of one thousand simulations for each energy level. The behaviour observed in the fifth energy level reflects the fact that, in this case, the terminal state is given by the fourth eigenstate $\chi_{4}(x)$. This is because $E_{4}$ is the closest energy eigenvalue to $E_{5}$ in the present example. To see this, we note that, if we write $\xi=\exp \left(\sigma \omega_{45} B_{t}-\frac{1}{2} \sigma^{2} \omega_{45}^{2} t\right)$ then as a consequence of (36) it follows that $H_{t}^{5}$ can be written in the form

$$
H_{t}^{5}=\frac{\xi\left(\pi_{4} E_{4}+\sum_{m}^{\prime} \pi_{m} E_{m} \exp \left(\sigma\left(\omega_{m 5}-\omega_{45}\right) B_{t}-\frac{1}{2} \sigma^{2}\left(\omega_{m 5}^{2}-\omega_{45}^{2}\right) t\right)\right)}{\xi\left(\pi_{4}+\sum_{m}^{\prime} \pi_{m} \exp \left(\sigma\left(\omega_{m 5}-\omega_{45}\right) B_{t}-\frac{1}{2} \sigma^{2}\left(\omega_{m 5}^{2}-\omega_{45}^{2}\right) t\right)\right)}
$$

where $\sum_{m}^{\prime}=\sum_{m \neq 4}$. Thus, the $\xi$-dependence cancels, and because $\omega_{m 5}^{2}-\omega_{45}^{2}>0$ for all $m \neq 4$, we see that as $t \rightarrow \infty$ the exponents in the denominator and the numerator go to zero and we are left with the leading term $E_{4}$.

In general, if the terminal value for the energy is chosen to be $E_{j}$ when the initial state $\psi_{0}(x)$ is orthogonal to $\chi_{j}(x)$, i.e. $\pi_{j}=0$, then the system necessarily relaxes into the eigenstate whose eigenvalue is the closest to $E_{j}$ (S. L. Adler, private communication). 
In Figure 4 we sketch the dynamics of the ensemble average for the probability density function for the location of the particle, in the case where the terminal state is the first excited state $\chi_{2}(x)$. This result corresponds to the average of a thousand examples of the type presented in Figure 2.

\section{RELAXATION TIMESCALE}

One of the advantages of the simulation methodology is that it opens up the possibility of a direct analysis of the timescale $\tau_{R}$ over which relaxation typically occurs. Let us define the process $M_{m j}$ by

$$
M_{m j}=\exp \left(\sigma \omega_{m j} B_{t}-\frac{1}{2} \sigma^{2} \omega_{m j}^{2} t\right),
$$

where for simplicity of notation we suppress the time dependence of $M_{m j}(t)$. The energy process (36) conditional on the outcome $E_{j}$ can then be written in the form

$$
H_{t}^{j}=\frac{\pi_{j} E_{j}+\sum_{m \neq j}^{\infty} \pi_{m} E_{m} M_{m j}}{\pi_{j}+\sum_{m \neq j}^{\infty} \pi_{m} M_{m j}},
$$

since $M_{j j}=1$. Clearly, if $M_{m j}$ is sufficiently small, then $H_{t}^{j}$ will approach its terminal value $E_{j}$ and the system will have relaxed. This occurs when $t>\tau_{R}$.

A version of the paper with figures can be downloaded from

http://theory.ic.ac.uk/ brody/DCB/sa8.pdf

FIG. 4. The average of $\rho_{t}(x)$ for a thousand runs. The initial and final conditions are given by $\psi_{0}(x)=\phi_{1}(x)$ and $\psi_{\infty}(x)=\chi_{2}(x)$, respectively, and we set $\alpha=2.5$. The $x$-axis is measured in units of $L$, the time axis is measured in units of $4 \mu^{2} L^{4} / \hbar^{4} \sigma^{2}$, and the magnitude of $\rho_{t}(x)$ is scaled by a factor of 1,000 . This plot illustrate how the density matrix is diagonalised.

In order to study the scale of $\tau_{R}$, we consider the probability that the process $M_{m j}$ is smaller than $\mathrm{e}^{-\lambda}$ for some number $\lambda$. If $\omega_{m j}>0$ this is given by

$$
\begin{aligned}
\mathbb{P}\left(M_{m j}<\mathrm{e}^{-\lambda}\right) & =\mathbb{P}\left(\sigma \omega_{m j} B_{t}-\frac{1}{2} \sigma^{2} \omega_{m j}^{2} t<-\lambda\right) \\
& =\mathbb{P}\left(B_{t}<\frac{1}{2} \sigma \omega_{m j} t-\frac{\lambda}{\sigma \omega_{m j}}\right) \\
& =N\left(\frac{1}{2} \sigma\left|\omega_{m j}\right| \sqrt{t}-\frac{\lambda}{\sigma\left|\omega_{m j}\right| \sqrt{t}}\right) .
\end{aligned}
$$


Here $N(x)$ is the standard normal distribution function

$$
N(x)=\frac{1}{\sqrt{2 \pi}} \int_{-\infty}^{x} \mathrm{e}^{-\frac{1}{2} y^{2}} \mathrm{~d} y .
$$

Note that in obtaining the second expression on the right-hand side of (48) we have used the fact that $\omega_{m j}>0$; whereas if $\omega_{m j}<0$ we would instead have the probability

$$
\mathbb{P}\left(M_{m j}<\mathrm{e}^{-\lambda}\right)=\mathbb{P}\left(B_{t}>\frac{1}{2} \sigma \omega_{m j} t-\frac{\lambda}{\sigma \omega_{m j}}\right) .
$$

However, owing to the symmetry relation $N(-x)=1-N(x)$ satisfied by the normal distribution function, the final result (48) is unaltered.

Now, for relaxation, we would like the probability (48) to be sufficiently great, say,

$$
\mathbb{P}\left(M_{m j}<\mathrm{e}^{-\lambda}\right) \geq 0.95
$$

for a suitably large $\lambda$. The choice of $\lambda$ corresponds to how far the reduction has to proceed for relaxation to have effectively set in. Because $N(x) \sim 0.95$ when $x \sim 1.65$, we can put a bound on the time variable such that the probability (48) is greater than 0.95 . This is given by

$$
\frac{1}{2} \sigma\left|\omega_{m j}\right| \sqrt{t}-\frac{\lambda}{\sigma\left|\omega_{m j}\right| \sqrt{t}}>1.65
$$

or equivalently

$$
\sqrt{t}>\frac{3.3+\sqrt{3.3^{2}+8 \lambda}}{2 \sigma\left|\omega_{m j}\right|} .
$$

In particular, for a fixed value of $j$, we would like this relation to hold for all values of $m \neq j$. That is to say, we would like $M_{m j}$ to become negligible for all $m \neq j$, which will ensure that $H_{t}^{j} \rightarrow E_{j}$. This can be guaranteed by choosing $m=j+1$, since this choice maximises the right hand side of (53). For example, if we take $\lambda=10$, then the relaxation timescale obtained is of the order

$$
\tau_{R} \sim \frac{40 \alpha^{4}}{\pi^{4} \sigma^{2}(2 j+1)^{2}}
$$

when the terminal state is $\chi_{j}(x)$. Thus provided $t \geq \tau_{R}$, we can be $95 \%$ confident that $M_{m j}<10^{-5}$ for every $m \neq j$.

We note, incidentally, that the result in (54) is based on the assumption that the expansion factor $\alpha$ is strictly and sufficiently greater than one. In the exceptional case where $\alpha=1$, we have $\tau_{R}=0$, which follows directly from (47) if one notes that $\pi_{m}=0$ for every $m \neq j$, and thus $H_{t}^{j}=E_{j}$ irrespective of the values of $M_{m j}$ and $t$.

When $\alpha \sim 1$, the foregoing analysis on the relaxation timescale needs to be carried out more carefully. For this purpose, let us consider the case in which we have $\alpha=1+\epsilon$, where the value of $\epsilon \geq 0$ can be very small. Now, if the system is initially in the ground state $\phi_{1}(x)$, then for $\epsilon \ll 1$, the transition probability $\pi_{m}(\epsilon)$ admits the expansion 


$$
\begin{aligned}
\pi_{m}(\epsilon) \sim & \frac{4 \sin ^{2}(\pi m)}{\pi^{2}\left(m^{2}-1\right)^{2}}+\left(\frac{16 \sin ^{2}(\pi m)}{\pi^{2}\left(m^{2}-1\right)^{3}}\right. \\
& \left.+\frac{4\left(3 \sin ^{2}(\pi m)-2 \pi m \sin (\pi m) \cos (\pi m)\right)}{\pi^{2}\left(m^{2}-1\right)^{2}}\right) \epsilon+\cdots
\end{aligned}
$$

for all $m$. In particular, $\pi_{1}(\epsilon)$ is of order one, whereas the next largest transition probability when $\alpha \sim 1$ is given, up to order $\epsilon^{2}$, by $\pi_{2}(\epsilon)=\frac{16}{9} \epsilon^{2}$. The implication of this is that the condition for $\pi_{m} M_{m 1}<\mathrm{e}^{-\lambda}$ to be valid for all $m \neq 1$ with $95 \%$ confidence-level is automatically satisfied for all $t \geq 0$, and thus it is not a sufficient criteria to determine the relaxation timescale.

Therefore, it is important in the small perturbation regime to determine how small $\epsilon$ can be for the $(p \times 100) \%$ confidence-level analysis to be viable. This can be determined if we replace $\lambda$ in (48) by $\lambda+\ln \pi_{m}(\epsilon)$ and 0.95 in (51) by $p$. The latter is equivalent to replacing 1.65 in (52) by $N^{-1}(p)$, where $N^{-1}(x)$ is the inverse of the normal distribution function (49). The result gives us

$$
\sqrt{t}>\frac{2 N^{-1}(p)+\sqrt{\left(2 N^{-1}(p)\right)^{2}+8\left(\lambda+\ln \pi_{m}(\epsilon)\right)}}{2 \sigma\left|\omega_{m j}\right|},
$$

and we maximise the right-hand side of (56) over all $m \neq j$, when $j=1$. The maximum is obtained by setting $m=2$, which leads us to the timescale for relaxation into ground state. This is given by

$$
\tau_{R} \sim \frac{1}{\sigma^{2} \omega_{21}^{2}}\left(N^{-1}(p)+\sqrt{2 \lambda+4 \ln (4 \epsilon / 3)+\left(N^{-1}(p)\right)^{2}}\right)^{2} .
$$

In particular, for a fixed $\epsilon$, either $\lambda$ or $p$ must be sufficient large so that the right-hand side of (57) is real. Conversely, for fixed $\lambda$ and $p$ the confidence-level analysis is viable only when $\epsilon$ is large enough to ensure the reality of the right-hand side of (57).

\section{TOWARDS THE QUANTUM ADIABATIC THEOREM}

The analysis of the relaxation timescale shows that for an infinitesimal expansion of potential well the initial eigenstate $\phi_{n}(x)$ will almost immediately relax into the corresponding eigenstate $\chi_{n}(x)$. This result suggests that when the potential well is expanded sufficiently slowly, then the dynamics of relaxation will force the system to remain in the $n$-th eigenstate. In other words, the stochastic evolutionary law appears to give rise to an analogue of quantum adiabatic theorem. In what follows we shall explore this idea in greater depth.

For this purpose we need to consider now the dynamics of a quantum system in the presence of a time-dependent Hamiltonian. Let us denote by $\hat{H}(t)$ a generic time-dependent Hamiltonian, and for each fixed time $t$ we write $\chi_{n}(t, x)(n=1,2, \ldots)$ for the $n$-th eigenstate of the operator $\hat{H}(t)$ with eigenvalue $E_{n}(t)$. Now, for an adiabatic approximation, we shall

assume that $\hat{H}(t), \chi_{n}(t, x)$, and $E_{n}(t)$ are continuous in $t$, and vary sufficiently slowly so that we can write

$$
\frac{\partial \chi_{n}(t, x)}{\partial t} \approx-\mathrm{i} E_{n}(t) \chi_{n}(t, x)
$$


Then for a general state $\psi_{t}(x)$ the Schrödinger evolution can be written as

$$
\frac{\partial \psi_{t}(x)}{\partial t}=-\mathrm{i} \hat{H}(t) \psi_{t}(x)=-\mathrm{i} \hat{H}(t) \sum_{n} a_{n}(t) \chi_{n}(t, x) .
$$

Therefore, we find that

$$
\sum_{n} \dot{a}_{n}(t) \chi_{n}(t, x)=0
$$

in the adiabatic approximation, from which it follows at once $\dot{a}_{k}(t)=0$. Thus the solution to the deterministic Schrödinger equation can be written as

$$
\psi_{t}(x)=\sum_{n} a_{n} \chi_{n}(t, x) .
$$

These relations are the consequences of the adiabatic approximation (58) in the deterministic unitary theory. What we would like to consider here is the implication of the assumption (58) in the case of the stochastic evolutionary law (9), which in the present context is given by

$$
\begin{aligned}
\mathrm{d} \psi_{t}(x)= & -\mathrm{i} \hat{H}(t) \psi_{t}(x) \mathrm{d} t-\frac{1}{8} \sigma^{2}\left(\hat{H}(t)-H_{t}\right)^{2} \psi_{t}(x) \mathrm{d} t \\
& +\frac{1}{2} \sigma\left(\hat{H}(t)-H_{t}\right) \psi_{t}(x) \mathrm{d} W_{t},
\end{aligned}
$$

where

$$
H_{t}=\frac{\int \psi_{t}^{*}(x) \hat{H}(t) \psi_{t}(x) \mathrm{d} x}{\int \psi_{t}^{*}(x) \psi_{t}(x) \mathrm{d} x}
$$

We note that (62) preserves the norm of $\psi_{t}(x)$. However, for the energy process $H_{t}$ we have

$$
\mathrm{d} H_{t}=\dot{H}_{t} \mathrm{~d} t+\sigma V_{t} \mathrm{~d} W_{t}
$$

instead of (12), where

$$
\dot{H}_{t}=\int \psi_{t}^{*}(x)\left(\partial_{t} \hat{H}(t)\right) \psi_{t}(x) \mathrm{d} x
$$

It is natural that the process corresponding to the expectation value of the Hamiltonian is no longer a martingale in the time-dependent case, but rather exhibits a drift, the sign of which depends on the expectation value of the time derivative of the Hamiltonian. Similarly for the variance process we obtain

$$
\begin{aligned}
\mathrm{d} V_{t}= & -\sigma^{2} V_{t}^{2} \mathrm{~d} t+\sigma \beta_{t} \mathrm{~d} W_{t} \\
& +2\left(\int \psi_{t}^{*}(x)\left(\hat{H}(t)-H_{t}\right)\left(\partial_{t} \hat{H}(t)-\dot{H}_{t}\right) \psi_{t}(x) \mathrm{d} x\right) \mathrm{d} t .
\end{aligned}
$$

Now, for state reduction, a necessarily condition is that the variance process $V_{t}$ is a supermartingale, i.e. on average a decreasing process. We observe that the last term in (66) 
is given by the covariance of the two operators $\hat{H}(t)$ and $\partial_{t} \hat{H}(t)$, which can be positive or negative. If it is negative, then the variance process $V_{t}$ is a supermartingale, whereas if it is positive, $V_{t}$ can still be a supermartingale, provided the covariance is not too large. In particular, if we write

$$
\Delta H_{t}=\sqrt{V_{t}}
$$

and

$$
\Delta \dot{H}_{t}=\sqrt{\operatorname{Var}\left[\partial_{t} \hat{H}(t)\right]}
$$

respectively for the standard deviations of $\hat{H}(t)$ and $\partial_{t} \hat{H}(t)$, then for the supermartingale condition we require

$$
2 \rho_{t} \Delta \dot{H}_{t} \Delta H_{t}<\sigma^{2}\left(\Delta H_{t}\right)^{4}
$$

where $\rho_{t}$ is the correlation between $\hat{H}(t)$ and $\partial_{t} \hat{H}(t)$. If we notice the fact that $-1 \leq \rho_{t} \leq 1$, we find, to ensure that $V_{t}$ is a supermartingale, it suffices that the following relation should hold:

$$
\frac{\Delta \dot{H}_{t}}{\Delta H_{t}}<\frac{1}{2} \sigma^{2}\left(\Delta H_{t}\right)^{2}
$$

In other words, we require the uncertainty in the change of $\hat{H}$ to be small compared with the uncertainty in $\hat{H}$ itself. Intuitively, if the change of the Hamiltonian is sufficiently slow, then $\partial_{t} \hat{H}(t)$ will be small, and therefore we would expect $\Delta \dot{H}_{t} \ll \Delta H_{t}$ be valid in the adiabatic regime. In particular, relation $(\overline{70})$ can be viewed as the consequence of adiabatic motion, which ensures that the variance $V_{t}$ is a supermartingale.

Now we turn to the the consideration of the stochastic equation (62). To begin with, let us define the process $\Pi_{t}^{k}$ by

$$
\Pi_{t}^{k}=\Pi_{0}^{k} \exp \left(\sigma \int_{0}^{t}\left(E_{k}(s)-H_{s}\right) \mathrm{d} W_{s}-\frac{1}{2} \sigma^{2} \int_{0}^{t}\left(E_{k}(s)-H_{s}\right)^{2} \mathrm{~d} s\right) .
$$

Then it is a straightforward exercise in Ito calculus to deduce that

$$
\mathrm{d} \Pi_{t}^{k}=\sigma\left(E_{k}(t)-H_{t}\right) \Pi_{t}^{k} \mathrm{~d} W_{t},
$$

from which it follows that the dynamics of the process

$$
a_{k t}=\left(\Pi_{t}^{k}\right)^{1 / 2}
$$

is given by

$$
\mathrm{d} a_{k t}=-\frac{1}{8} \sigma^{2}\left(E_{k}(t)-H_{t}\right)^{2} a_{k t} \mathrm{~d} t+\frac{1}{2}\left(E_{k}(t)-H_{t}\right) a_{k t} \mathrm{~d} W_{t},
$$

Therefore, if we define

$$
\psi_{t}(x)=\sum_{j} a_{k t} \chi_{j}(t, x)
$$


where the functions $\chi_{j}(t, x)$ are eigenfunctions of $\hat{H}(t)$, then we obtain

$$
\mathrm{d} \psi_{t}(x)=\sum_{k}\left(\mathrm{~d} a_{k t}\right) \chi_{k}(t, x)+\sum_{k} a_{k t}\left(\partial_{t} \chi_{k}(t, x)\right)
$$

and thus

$$
\begin{aligned}
\mathrm{d} \psi_{t}(x)= & -\mathrm{i} \hat{H}(t) \psi_{t}(x) \mathrm{d} t-\frac{1}{8} \sigma^{2}\left(\hat{H}(t)-H_{t}\right)^{2} \psi_{t}(x) \mathrm{d} t \\
& +\frac{1}{2} \sigma\left(\hat{H}(t)-H_{t}\right) \psi_{t}(x) \mathrm{d} W_{t} \\
& +\left(\sum_{k} a_{k t}\left(\partial_{t} \chi_{k}(t, x)-\hat{H}(t) \chi_{k}(t, x)\right)\right) \mathrm{d} t .
\end{aligned}
$$

Thus far our analysis is exact. Now, in the adiabatic regime, we have the relation (58), which implies that

$$
\left(\partial_{t} \chi_{k}(t, x) \approx \hat{H}(t) \chi_{k}(t, x)\right.
$$

Therefore, substituting this relation into equation (77), we see that in the adiabatic approximation, the process $\psi_{t}(x)$ defined by $(75)$ is the solution to the stochastic dynamics (62).

We observe that in the adiabatic approximation the process $\Pi_{t}^{k}$ has the interpretation that it represents the probability that $\psi_{t}(x)$ is in the eigenstate $\chi_{k}(t, x)$. On the other hand, $\Pi_{t}^{k}$ is also a martingale, satisfying (72). Thus the expected probability that $\psi_{t}(x)$ is in the state $\chi_{k}(t, x)$, given information up to time $s$, is precisely the probability that $\psi_{s}(x)$ is in the state $\chi_{k}(s, x)$. It follows, therefore, that if $\psi_{t}(x)$ is in the state $\chi_{k}(0, x)$ at time 0 , it will be in the state $\chi_{k}(t, x)$ at time $t$ with probability one.

\section{DISCUSSION}

Although in the present paper we have primarily examined the square-well potential, many of the results obtained, namely, the wave function (31), the conditional Hamiltonian process (36), the probability distribution of the particle (37), and the bound on relaxation time (53), are independent of the specific model being considered. As long as the eigenvalues $E_{m}$ and the eigenfunctions $\chi_{m}(x)$ of the Hamiltonian are known, either analytically or numerically, these results can be applied to study the details of the dynamics. In other words, the general approach outlined here, based on the nonlinear filtering methodology introduced in Brody \& Hughston (2002), can be applied to study a wide range of problems in perturbation theory, including of course a more generic time-dependent Hamiltonian. We hope to take up this line of investigation in greater detail elsewhere.

The point of view we have put forward in this paper is that the dynamical law (9) offers a simple but plausible characterisation of the subsequent evolution of a quantum system after the Hamiltonian has been perturbed. If a measurement of the system energy is carried out after a passage of time greater than the relaxation timescale, then according to the stochastic postulate, when the system is initially in the eigenstate $\phi_{n}(x)$, the eigenvalue $E_{m}$ will be observed with probability $\pi_{n m}$. This is because the system is already in the eigenstate $\chi_{m}(x)$ 
with that probability. On the other hand, according to the unitarity postulate associated with the Schrödinger equation, the system is in a superposition of a myriad of eigenstates. Nevertheless, the outcome of the energy measurement will give $E_{m}$ with probability $\pi_{n m}$. The question thus arising is whether one can distinguish the two theories by means of a suitable experiment. To this end, we note that, for time $t \geq \tau_{R}$, the state of the system, according to the stochastic law, is given by a mixed-state density matrix, whose diagonal elements, in the energy basis, are given by $\pi_{m}=\pi_{n m}$; whereas according to unitary law, the system is in a pure state, given by the superposition of the energy eigenstates, whose coefficients are given by $\sqrt{\pi_{m}}$. Therefore, if these pure and mixed states can be distinguished statistically in some way by means of experiments, then in principle one can rule out at least one of the two postulates indicated here. At present, the possibility of distinguishing the two remains an open problem.

The authors are grateful to S. L. Adler, J. Anandan, A. Bassi, T. A. Brun, I. Buckley, and B. K. Meister for stimulating discussions. DCB acknowledges support from The Royal Society. LPH acknowledges the support and hospitality of the Institute for Advanced Study, where part of this work was carried out.

[1] Adler, S. L., Brody, D. C., Brun, T. A. \& Hughston, L. P. 2001 J. Phys. A 34, 8795.

[2] Adler, S. L. \& Horwitz, L. P. 2000 J. Math. Phys. A: Math Gen. 41, 2485.

[3] Adler, S. L., 2002 J. Phys. A: Math Gen. 35, 841.

[4] Bassi, A. \& Ghirardi, G. C. 2002 Preprint quant-ph/0201122.

[5] Bedford, D. \& Wang, D. 1975 Nuo. Cim. B26, 313.

[6] Bedford, D. \& Wang, D. 1977 Nuo. Cim. B37, 55.

[7] Bender, C. M., Brody, D. C. \& Meister, B. K. 2000 J. Phys. A: Math. Gen. 33, 4427.

[8] Brody, D. C. \& Hughston, L. P. 2002 Proc. R. Soc. London A 458, 1117.

[9] Brody, D. C. \& Hughston, L. P. 2002 J. Math. Phys. 43 (to appear, quant-ph/0203035).

[10] Diosi, L. 1988 J. Phys. A21, 2885.

[11] Doob, J. L. 1942 Ann. Math. 43, 351.

[12] Ghirardi, G. C., Rimini, A. \& Weber, T. 1986 Phys. Rev. D 34, 470.

[13] Ghirardi, G. C., Pearle, P. \& Rimini, A. 1990 Phys. Rev. A 42, 78.

[14] Gisin, N. 1984 Phys. Rev. Lett. 52, 1657.

[15] Gisin, N. 1989 Helv. Phys. Acta 62, 363.

[16] Hughston, L. P. 1996 Proc. R. Soc. London A 452, 953.

[17] Liptser, R. S. \& Shiryaev, A. N. 1974 Statistika sluchainykh protsessov (Moscow: Nauka) (English translation by Wilson S S 2000 Statistics of Random Processes Vol. I and II, 2nd ed. Berlin: Springer).

[18] Pearle, P. 2000 in Open Systems and Measurement in Relativistic Quantum Theory, H.P. Breuer and F. Petruccione, eds. Berlin: Springer.

[19] Percival, I. 1994 Proc. R. Soc. London A 447, 189.

[20] Percival, I. 1995 Proc. R. Soc. London A 451, 503.

[21] Percival, I. 1998 Quantum State Diffusion Cambridge: Cambridge University Press. 